\title{
Vision-based Lawn Boundary Recognition for Mowing Robot
}

\author{
Yao Guo, Fu-Chun Sun \\ Department of Computer Science and Technology, Tsinghua University \\ State Key Lab. of Intelligent Technology and Systems, \\ Tsinghua National Laboratory for Information Science and Technology (TNList), BeiJing, China \\ E-mail: guoyao14@mails.tsinghua.edu.cn, fcsun@mail.tsinghua.edu.cn
}

\begin{abstract}
We present a novel method for mowing robot's lawn boundary recognition based on Gabor filters and support vector machine (SVM). Robust texture features of images are extracted and concatenated using Gabor filters. The principle components analysis (PCA) approach is then used to reduce the dimensionality of Gabor features. Based on the compressed features, SVM model is trained and used to perform the grass texture classification task. The boundary of lawn is then recognized according to the ratio of grass area of the image. To demonstrate the effectiveness and robustness of our proposed method, a dataset is created with about 1500 images of different lawn scenes. Result shows that a classification accuracy of $96.7 \%$ can be reached when SVM is used. Experiments of the lawn boundary recognition have also been conducted on the mowing robot under different lighting conditions. The recognition rate tested is $98.3 \%$, which proves the efficiency and superiority of our proposed method.
\end{abstract}

Keywords-component; mowing robot; computer vision; gabor filter; PCA; SVM

\section{INTRODUCTION}

Mowing robot is a kind of mobile robot which can mow the lawn autonomously. Compared with manual mowers, mowing robot has the superiority of labor-saving and usersafety. For the past decades, mowing robot acquires remarkable development driven by the huge demand. For security factors, mowing robot should be restricted within the lawn while it is working, so boundary recognition becomes an essential ability of the mowing robot. Most mowing robots are equipped with a kind of boundaryrecognition system called "electronic fence" [1]. An electronic fence consists of a circle of wire surrounding the boundary and a magnetometer on the mowing robot. The magnetometer monitors the intensity of magnetic field, the intensity value will increase rapidly when the mowing robot reaches lawn boundary. The method of electronic fence is cumbersome as the wire need to be setted every time before the robot start working. To improve the performance of the lawn boundary recognition, vision-based methods have been researched in recent years.

Lu et al [2] applied the method of image segmentation to distinguish the lawn from background and extract the boundary line. In this method, the threshold range of HSI value is setted to filter the background cursorily. The image is then converted to grayscale, and texture feature matrix of the grass is extracted from the image. The texture feature matrix is applied to search for matches from the image, so that a binary image is generated. Furtherly, by extracting the histogram of the binary image and computing the histogram's peak, remaining components of the background is finally removed. Although the results show that this method can reach $70 \%$ recognition rate, the recognition rate is not high enough for the practical application, and there is a lack of robustness for environmental factors such as lighting conditions.

Miao et al [3] proposed a method of lawn boundary recognition by calculating the ratio of grass area of the image. From this method, the image is divided into 9 grids. By computing the ratio of grass area in each grid and applying an optimal judgement criteria, the method can judge whether the mowing robot is getting out of boundary. The results show that the method can reach 95\% recognition rate when tested on the mowing robot. However, there still exists the risk of misrecognition against complex background.

Zhu [4] used Gabor filters and Relevance Vector Machine (RVM) to determine the surface types. This method performs surface features extraction with fewest number of image features nodes and Gabor wavelet scales direction. RVM neural-based approach to surface type recognition is then proposed. The results show that the recognition rate of Gabor-RVM method with $91.9 \%$ can be achieved. Also, contrast experiments show that Gabor filters perform better than other methods in feature extraction. However, the recognition rate still remains to improve for practical application.

In this paper, we apply Gabor filters to extract robust features from lawn scenes, as Gabor filters are successfully practiced in the fields of texture classification [5][6][7] and object recognition [8][9], which is confirmed to have a better performance than other descriptors like LBP. Consider that Gabor features are of so high dimensionality that it is impractical to use Gabor features directly, we proposed PCA methods [10] to compress the feature and extract components that have better classification performance. SVM method with Radial Basis Function (RBF) kernel is applied to perform texture classification [11]. Finally, lawn boundary is recognized according to the grass area ratio in the real-time image, much faster than state-of-art deeplearning methods.

The remainder of the paper is organized as follows: In Section 2, we illustrate how we construct our dataset, then we briefly outline the method of extracting grass textures with Gabor filters, followed by performing the texture classification with SVM. In Section 3, we present and analysis our experiments conducted on the dataset and 
mowing robot. Section 4 draws a conclusion and illustrate the future work of our research.

\section{SYSTEM DESCRIPTION}

This section mainly describes the method of lawn boundary recognition, which consists of the major components including dataset construction, extraction and classification of grass textures, boundary recognition. Brief illustration of these components are provided in the subsequent sections.

\section{A. Dataset Construction}

To ensure the robustness of our proposed method, it is critical to take environmental factors into consideration while constructing the dataset, including lighting condition, scale factors and orientation variance. The dataset consists of images captured by a camera fixed on the mowing robot. While the robot is moving within the lawn, the camera keeps capturing images with the rate of $24 \mathrm{fps}$. Finally about 1500 images of lawn scenes are captured under different lighting and weather conditions in morning, noon and dusk. Images are of size $640 * 480$ pixels as Fig 1 shows. Then each image is converted to grayscale and is divided into small images of $40 * 40$ pixels for feature extraction and classification, as Fig 2 shows.
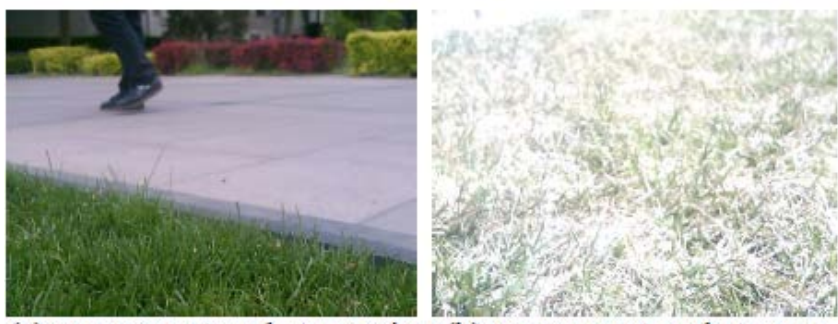

(a) scenes captured at morning (b) scenes captured at noon

Figure 1. Dataset consists of lawn scenes captured under different environment conditions
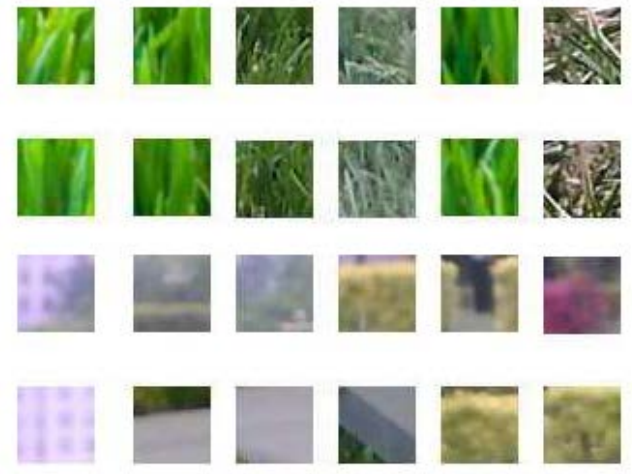

\section{Others}

Figure 2.

Small patches for feature extraction and texture classification

\section{B. Feature Extraction of Lawn Scenes}

Due to the change of lighting condition in unstructured environment, it is impractical to apply color features in boundary recognition. In this paper, we apply Gabor filters to extract robust features corresponding to the texture properties of grass. The process of feature extraction is divided into three steps as Fig 3 shows. We first filter each image with Gabor filters. Then we applied PCA methods to reduce the dimensionality of features. Finally L2 normalization is performed to achieve better classification results. Details of the above steps are illustrated in following sections.

- Gabor filters: Gabor filters have been found to be particularly appropriate for texture representation and discrimination, as Gabor filters are sensitive to spatial locality, orientation selectivity, and spatial frequency. It is also confirmed that frequency and orientation representations of Gabor filters are similar to those of the human visual system. So we proposed Gabor filters to extract features of lawn scenes. A 2D Gabor filter is defined by a complex exponential function multiplied by a Gaussian function, and the function can be expressed as

$$
g(x, y ; \lambda, \theta, \sigma, \gamma)=\exp \left(\frac{-x^{2}+y^{2}}{2 \sigma^{2}}\right) \exp \left(i\left(2 \pi \frac{x}{\lambda}+\psi\right)\right)
$$

Where

$$
\begin{gathered}
x^{\prime}=x \cos \theta+y \sin \theta \\
y^{\prime}=-x \sin \theta+y \cos \theta
\end{gathered}
$$

The parameter $\lambda$ represents the wavelength, $\theta$ indicates the orientation, $\sigma$ represents scale factor at orthogonal direction, $\gamma=\frac{\lambda}{\theta}$ is applied to ensure that a change of $\sigma$ could correspond to the scale change in the Gabor filter.

A set of parameters $(\theta, \sigma, \gamma)$ are chosen to generate multi Gabor kernels. Examples of different Gabor kernels are illustrated in Fig 4. During our experiments, we set the parameters as follows: $\gamma=45, \theta=\{0,22,36,45,54,72\}$ degree, $\sigma=\{1,2,4\}$. So there are 18 Gabor filters generated in total. By convolving an image with a Gabor filter, a response is obtained with 1600 elements. By concatenating the 18 filter responses, the pattern vector has a dimensionality of $1600 * 18=28,800$. Fig 5 shows Gabor filters responses of grass textures.

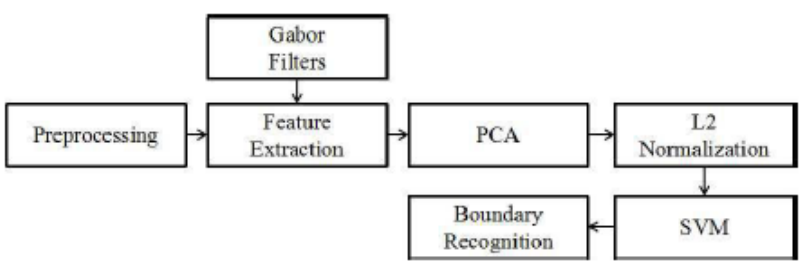

Figure 3 Process of feature extraction and classification 

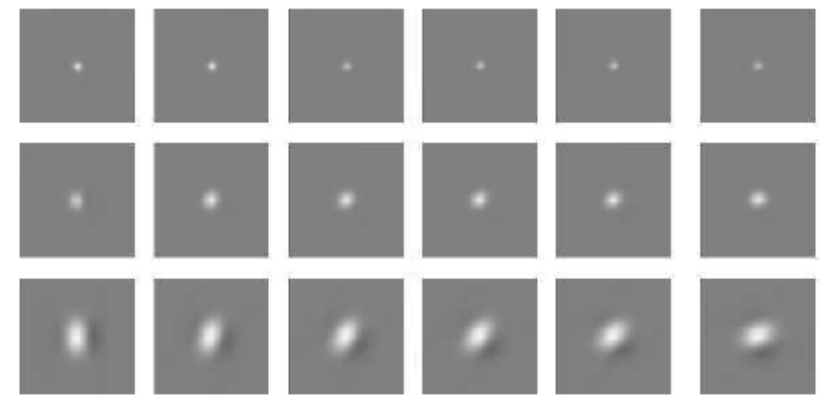

Figure 4. Gabor kernels with different parameters
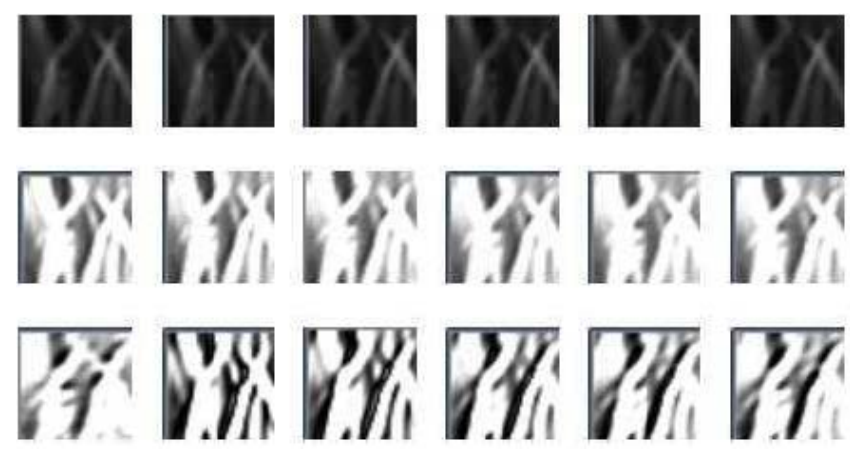

Figure 5. Gabor responses of grass textures

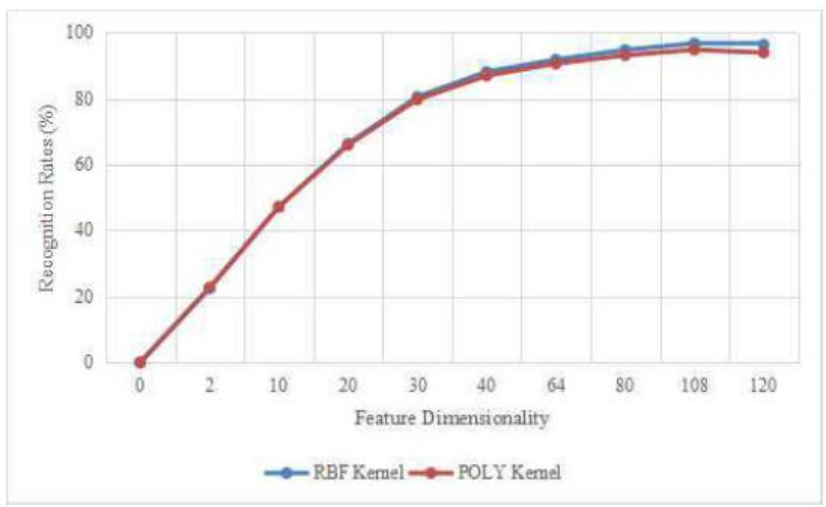

Figure 6 Recognition rates with different feature dimensionality

- PCA methods: The method of Principle Component Analysis (PCA) aims to find a set of orthonormal vectors in the data space, which can help reduce the dimensionality of the feature vector and maximize the feature's variance, therefore the classifier can perform better both in speed and accuracy.

Consider a dataset with $\mathrm{N}$ samples $x_{i} \in R^{D}$, $i=(1,2 \ldots n)$ in total, each sample is of $\mathrm{D}$ dimensions. Scatter matrix $S_{T} \in R^{D^{* D}}$ is defined as

$$
S_{T}=\sum_{i=1}^{N}\left(x_{i}-\mu\right)\left(x_{i}-\mu\right)^{T}=A A^{T}
$$

where $\mu$ is the mean image of the dataset.

$$
A=\left[x_{1}-\mu \ldots x_{N}-\mu\right]
$$

The dimensions of matrix $S_{T}$ is too huge to perform an computation directly. Instead, we obtain the eigenvector $V_{P C A}$ by solving the expression $R V_{P C A}=V_{P C A} \Lambda$, while $R=A^{T} A, \Lambda=\left[\lambda_{1 . . .} \lambda_{P}\right]$ is the eigenvector of $\mathrm{R}$, components in $\Lambda$ are in descending order, and $\mathrm{P}$ is the dimension of the new feature after PCA operation. We then construct the PCA subspace $W_{P C A}$ by multiplying matrix A with eigenvectors $V_{P C A}$. Finally, we can compute the new feature vector $\mathrm{y}$ of image $\mathrm{x}$ in the PCA subspace $W_{P C A}$, the expression of projection is shown below

$$
y=W_{P C A}^{T}(x-\mu) \in R^{P}
$$

- $\quad$ L2 normalization: We then perform normalization to the features after PCA operation to restrict the range of feature values, so that the process of convergence could be accelerated and the capability of classifier is optimized. This method applies the root sum square (L2 norm) of the feature vector to perform normalization. Consider an feature vector $x=\left[n_{1} \ldots n_{d}\right]$, the process of L2 normalization is shown as below

$$
\begin{gathered}
\operatorname{Norm}(X)=\sqrt{\sum_{i=1}^{D} x_{i}^{2}} \\
X^{\prime}=\left[\frac{n_{1}}{\operatorname{Norm}(X)} \ldots \frac{n_{d}}{\operatorname{Norm}(X)}\right]
\end{gathered}
$$

\section{Lawn Texture Classification}

We apply Support Vector Machine (SVM) to perform the texture classification of lawn scenes. SVM is a classifier aimed at solving two-class problems by computing an optimal hyperplane. It is widely used in machine learning due to its superiority in handling non-linear classification problems. One non-linear SVM classifier applies an nonlinear operator $\phi(\mathrm{x})$ to map the input data $\left(\mathrm{x}_{\mathrm{i}}, \mathrm{y}_{\mathrm{i}}\right)$ into a higher dimensional feature space, where an optimal hyperplane could be found as a decision surface. The expression of SVM with the kernel function is found to be.

$$
g(x)=\operatorname{sgn}\left(\sum_{i} y_{i} \alpha_{i} K\left(x_{i}, x\right)+b\right)
$$

where sgn is the sign function, $x_{\mathrm{i}}$ represents the support vector learned by SVM, $K\left(x_{i}, x\right)=\phi\left(x_{i}\right)^{T} \phi(x)$ is the kernel function. In this research, we apply Radial Basis Function (RBF) kernel and Polynomial (POLY) kernel to perform 
SVM training and classification. The RBF kernel and POLY kernel is defined as follows

$$
\begin{gathered}
K_{R B F}\left(x_{i}, x\right)=\exp \left(-\gamma\left\|x_{i}-x\right\|^{2}\right), \gamma>0 \\
K_{P O L Y}\left(x_{i}, x\right)=\left(x_{i} x+1\right)^{d}
\end{gathered}
$$

where $\gamma=0.51$ in RBF kernel and $d=3.43$ in POLY kernel. The coefficients $\alpha_{i}$ and $b$ can be computed by the following quadratic programming problem

$$
\begin{gathered}
\max \left[\sum_{i} \alpha_{i}-\frac{1}{2} \alpha_{i} \alpha_{j} y_{i} y_{j} K\left(x_{i}, x_{j}\right)\right] \\
\text { s.t. } \sum_{i} \alpha_{i} y_{i}=0,0<\alpha_{i}<C
\end{gathered}
$$

where $C$ is the penalty factor aimed to maximize the margin and avoid overfitting. In this research we set $C=12.50$, which is proven to be optimal by the iterative method.

\section{Lawn Boundary Recognition}

We perform lawn boundary recognition on the basis of the ratio of grass area $\eta$ in the image. Each image is divided into grids of $40 * 40$ pixels, so there are totally 192 grids in one image. Each single grid is classified as grass or nongrass by SVM and a following criterion

$$
h(i, j)=\operatorname{sgn}\left(n_{i+1}-n_{\min }\right)
$$

Where $h(i, j)$ is a sign function to judge whether a grid at position $(i, j)$ is grass or not, which help filter out the false accepted grids after SVM prediction. $n_{i+1}$ is the number of grass grids in the beneath row, and we choose $n_{\min }=3$ as the threshold. To determine the status of the mowing robot, we propose the threshold of grass area ratio $T_{B}$ and $T_{O}$, where $T_{B}=20 \%$ indicates that the robot reaches the lawn boundary, and $T_{O}=5 \%$ represents the threshold that the robot gets out of the lawn. Thresholds are adjusted due to the speed $v$ of mowing robot and the height $h$ of camera.

\section{RESULTS AND ANALYSIS}

The dataset of lawn scenes is divided into training set and testing set. The training set consists of 640 images, 140 of those are of grass category, the rest are of non-grass category. The testing set consists of 320 images, 120 of those are of grass category, while the rest are of non-grass category. All of those images are first filtered with Gabor filters. The data of 18 Gabor responses are concatenated by the rows to form a feature vector with a dimensionality of 28,800 . PCA method is then applied to reduce the high dimensionality of feature vector and optimal the classification performance. The size of the feature vector is reduced to 108 after PCA process. Then we apply SVM ( $\gamma=0.51, C=12.50)$ to perform classification of grass textures. POLY kernel is used to make a comparison with RBF method.

We have implemented our algorithms on the Jetson TK1 embedded hardware, which is of strong capability to perform lawn texture classification and boundary recognition.

Fig 6 shows the lawn texture recognition rates of different methods adapted in this research. The highest recognition rates with RBF kernel and POLY kernel are $96.7 \%, 94.8 \%$, while the dimensionality of feature vectors is 108. Results in Fig 6 and Table 1 have shown that RBF kernel performs better than POLY kernel both in time consumption and recognition rates. Compared with state-ofart recognition methods referred above, Gabor-PCARBFSVM method has outstanding performance in accuracy and robustness.

TABLE I. CLASSIFICATION RESULT OF THE LAWN DATASET

\begin{tabular}{|l|c|c|}
\hline \multirow{2}{*}{\multicolumn{1}{|c|}{ Results }} & \multicolumn{2}{|c|}{ Methods } \\
\cline { 2 - 3 } & RBF-SVM & POLY-SVM \\
\hline Feature of Dimension & 108 & 108 \\
\hline Number of SVs & 92 & 126 \\
\hline SVM Training Time (s) & 13 & 72 \\
\hline Recognition Rate (\%) & 96.7 & 94.8 \\
\hline True Positive Rate (\%) & 98.3 & 96.7 \\
\hline False Negative Rate (\%) & 95.0 & 93.3 \\
\hline
\end{tabular}

We then conduct a series of boundary recognition experiments on the mowing robot. During these experiments, the mowing robot is moving at a speed of $0.5 \mathrm{~m} / \mathrm{s}$ on a lawn with the size of $20 * 15 \mathrm{~m}$. SVM model is pre-trained and used to perform recognition on the images captured by the camera. The robot is setted to process 1 frame per-second. To confirm the robustness of our system, we conduct experiments in different weather and lighting condition. Fig 7 illustrates the results of mowing robot's lawn boundary recognition.

In order to display the recognition process of the system, grids classified as grass are marked as white to make the result visible. In Fig 7 (a), the mowing robot is moving within the lawn, while it is about to get out of the lawn in Fig 7 (c),where the ratio $\eta$ of lawn area in is $40.6 \%$ in Fig 7 (b) and $17.7 \%$ in Fig 7 (d). During the experiments, the mowing robot has reached lawn boundary for 120 times in total, but only gets out of the lawn for 2 times, results have shown that the recognition rate of lawn boundary is $98.3 \%$, which proves the efficiency of the Gabor-PCA-RBFSVM method we have proposed. 

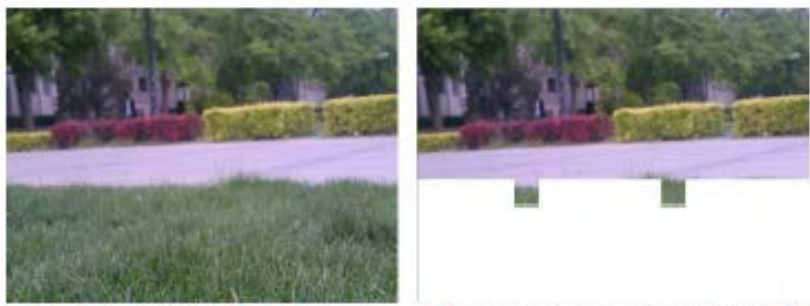

(a) robot within the lawn area

(b) grass area ratio is $40.6 \%$
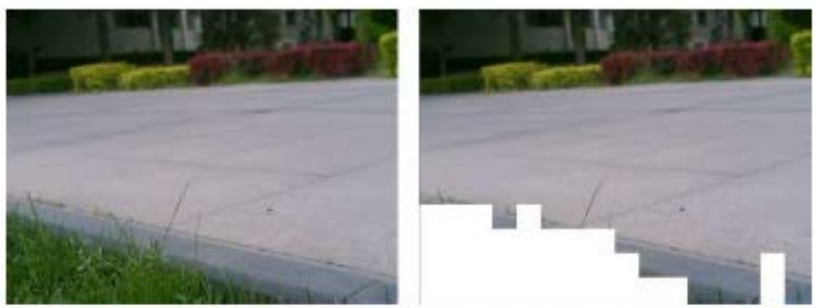

(c) robot at the lawn boundary
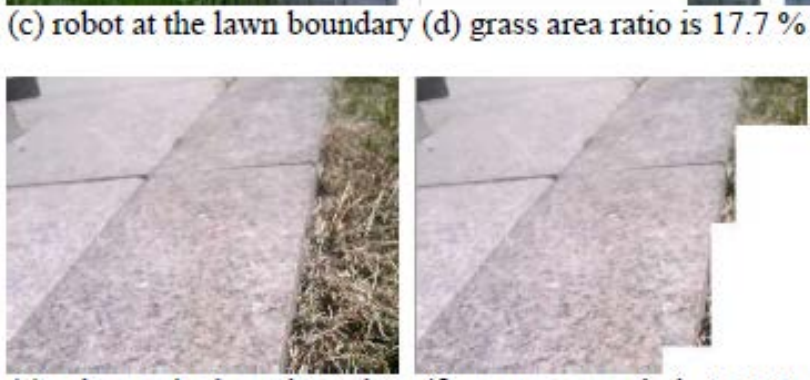

(e) robot at the lawn boundary (f) grass area ratio is $18.9 \%$

Figure 7. Results of lawn boundary recognition on the mowing robot.

\section{CONCLUSION AND FUTURE-WORKS}

In this research, we proposed a vision-based lawn boundary recognition system to take place of the "electronic fence" applied in state-of-art mowing robot. Out method applies Gabor filters and Support Vector Machine to perform lawn texture classification and boundary recognition. Results of SVM prediction have confirm the efficiency and robustness of our proposed method. Additionally, we have implemented our recognition system on the mowing robot and performed experiments on the lawn, and recognition rates have proven the superiority of our system.
However, there still exists some problems to be solved. For example, camera shake may happen while the mowing robot is moving on the uneven ground. Therefore, misclassification may occur when the images get ambiguous. In future work, we shall apply Convolutional Neural Network (CNN) to perform a lawn scene cognition and try to minimize the influence of the camera shake. We will also extend our system to vision-based lawn obstacle recognition and path planning, which strengthens the capability of the mowing robot in handling more complex task.

\section{REFERENCES}

[1] Ehud Peless, Shai Abrahamson, and Ilan Peleg, "Method for operating a robot," Google Patents, Dec. 10, 2002, US Patent 6,493,613.

[2] Xi Lu, Yu Liu, Huijiang Du, and Shixin Xu, "The uncut lawn cognition algorithm based on image analysis," in Intelligent Control and Automation (WCICA), $201411^{\text {th }}$ World Congress on. IEEE, 2014, pp. 5138-5142.

[3] Liang Zhao, Meng Su, and Yun Chen Miao, "High accuracy analysis of scara industrial robot based on screw theory," in Applied Mechanics and Materials. Trans Tech Publ, 2012, vol. 130, pp. 249255.

[4] Jiang Zhu, Yaonan Wang, Hongshan Yu, Wenge Wang, and Yaxing Wen, "Sensing incline terrain for mobile robot autonomous navigation under unknown environment,” pp. 2296-2301, 2010.

[5] S Arivazhagan and L Ganesan, "Texture classification using wavelet transform," Pattern recognition letters, vol. 24, no. 9, pp. 1513-1521, 2003.

[6] Lin Zhang, Zhiqiang Zhou, and Hongyu Li, "Binary gabor pattern: An efficient and robust descriptor for texture classification,” pp. 81 -84, 2012.

[7] Zhi-Chun Huang, Patrick PK Chan, Wing WY Ng, and Daniel S Yeung, "Content-based image retrieval using color moment and gabor texture feature," vol. 2, pp. 719-724, 2010.

[8] Xiaohui Shen and Ying Wu, "A unified approach to salient object detection via low rank matrix recovery," in Computer Vision and Pattern Recognition (CVPR), 2012 IEEE Conference on. IEEE, 2012, pp. 853-860.Article in a conference proceedings.

[9] SHEN Lin-Lin and JI Zhen, "Gabor wavelet selection and svm classification for object recognition," Acta Automatica Sinica, vol. 35, no. 4, pp. 350-355, 2009.

[10] Ergun Gumus, Niyazi Kilic, Ahmet Sertbas, and Osman N Ucan, "Evaluation of face recognition techniques using pca, wavelets and svm,” Expert Systems with Applications, vol. 37, no. 9, pp. 64046408, 2010.

[11] Shu Liao, Max WK Law, and Albert Chung, "Dominant local binary patterns for texture classification," Image Processing, IEEE Transactions on, vol. 18, no. 5, pp. 1107-1118, 2009. 